\title{
Energy Efficient Clustering for Wireless Sensor Networks using EASSR
}

\author{
Y. Chalapathi Rao*1, Ch. Santhi Rani ${ }^{2}$ \\ ${ }^{1}$ Dept. of ECE, Anurag Engineering College, Kodad, Telangana, India \\ ${ }^{2}$ Dept. of ECE, DMSSVH College of Engineering, Machalipatnam, India \\ ${ }^{*}$ Corresponding author, e-mail: chalu.8421@gmail.com ${ }^{1}$, santhirani.ece@gmail.com²
}

\begin{abstract}
Wireless Sensor Networks (WSNs) consist of a large quantity of small and low cost sensor nodes powered by small non rechargeable batteries and furnish with various sensing devices. The cluster-based technique is one of the good perspectives to reduce energy consumption in WSNs. The lifetime of WSNs is maximized by using the uniform cluster location and balancing the network loading between the clusters. We have reviewed various energy efficient schemes apply in WSNs of which we concerted on clustering approach. So, in this paper we have discussed about few existing energy efficient clustering techniques and proposed an Energy Aware Sleep Scheduling Routing (EASSR) scheme for WSN in which some nodes are usually put to sleep to conserve energy, and this helps to prolong the network lifetime. EASSR selects a node as a cluster head if its residual energy is more than system average energy and have low energy consumption rate in existing round. The efforts of this scheme are, increase of network stability period, and minimize loss of sensed data. Performance analysis and compared statistic results show that EASSR has significant improvement over existing methods LEACH, SEP, E-SEP and M-GEAR protocol in terms of energy consumption, network lifetime and data units gathered at $B S$.
\end{abstract}

Keywords: EASSR, LEACH, M-GEAR, SEP, WSNS

Copyright $\odot 2015$ Institute of Advanced Engineering and Science. All rights reserved.

\section{Introduction}

Wireless Sensor Network (WSN) due to its potentially large application area emerged as a premier research topic. WSN consists of thousands tiny nodes which individually has limited capabilities but collectively they can form a very useful network for various applications like environment monitoring, disaster management, vehicle tracking, habitat monitoring etc. Usually the WSN once deployed, works in an unattended manner and each sensor node has limited battery capacity. So after each operation a node comes closer to death which finally brings the whole WSN's operation to a halt. So energy is the main constraint for any application using WSN.

Wireless Sensor Networks (WSNs) [1] consist of a number of sensor nodes with sensing, computation, and wireless communications capabilities. The sensor nodes senses data or gather information about the events occurring near that sensor, then the sensor nodes processes those data gathered and at last those data are transmitted to other sensor nodes or base stations if required and also a sensor node can receive data from other sensor nodes. This way the sensor nodes create a network and communicate with each other nodes in that network. A WSN contains hundreds or thousands of sensor nodes. These sensors have the ability to communicate either among each other or directly to an external base-station (BS). A greater number of sensors allows for sensing over larger geographical regions with greater accuracy.

In a large scale WSN the nodes which are nearer to sink are always used for forwarding packet from all other distant nodes. Due to this, the nodes which are nearer to sink are out of energy very soon and an energy hole is created near the sink and the sink becomes unreachable, while maximum nodes in the network are still alive [2]. Sensor node are densely deployed in wireless sensor network that means physical environment would produce very similar data in close by sensor node and transmitting such type of data is more or less redundant. If sensor nodes of same application and at minimum distance between them 
alternatively perform data collecting, processing and communication then we can able to transmit information to the base station for longer time. Thus network lifetime will be improved.

The main objective of this paper is to develop new approaches for providing energy efficiency, longer lifetime, and quick data delivery for WSNs which are mainly used for those areas where nodes remaining largely inactive for long periods of time, but then becoming suddenly active when something is detected. A detailed literature survey is done for developing an idea about the solutions already provided for these problems. This paper studies the performances of some existing algorithms and proposes an efficient algorithm for fulfilling its objective. A solution to the above mentioned problems is proposed by putting some nodes in sleep mode, whereas the other nodes are kept in active mode for sensing and communication tasks. Sensor nodes are alternatively sense the environment and perform data collection and processing.

EASSR protocol is aimed at prolonging the lifetime of the sensor networks by balancing the energy consumption of the nodes. It makes the high residual energy node to become a cluster-head. The nodes are used to collect the energy information of the nearest sensor nodes and tally with system average energy, and then select the cluster-heads. Our proposed algorithm compared with some of the existing energy efficient routing protocols to assess performance.

Various literatures present different ways for handling challenges and issues in WSN. Some of them propose energy harvesting in sensor nodes using solar panel. Periodic sleeping of nodes, energy efficient MAC protocol and energy efficient routing, fault tolerant routing, deployment of multiple sink are some of such proposals that can be found in literatures. In the present work, in order to handle challenges, energy efficient adaptive dynamic clustering technique is deployed. This approaches adapted here to overcome the challenges.

In order to support data aggregation through efficient network organization, nodes can be partitioned into a number of small groups called clusters. Clustering is a key technique used to extend the lifetime of sensor networks [3]. Clustering makes the network scalable and thus reduces energy consumption of the nodes. Clustering is a good approach to achieve an enhanced lifetime of wireless sensor networks. So far, many clustering technique have been introduced. In clustering the nodes are grouped into small cluster regions. The leader node of a cluster region is referred to as cluster head $(\mathrm{CH})$. All nodes in a cluster transmit their sensed information to corresponding $\mathrm{CH}$. $\mathrm{CH}$ manages the group communication with the BS [4].

Clustering results in a two-tier hierarchy in which cluster heads $(\mathrm{CHs})$ form the higher tier while member nodes form the lower tier. The member nodes report their data to the respective $\mathrm{CHs}$. The $\mathrm{CHs}$ aggregate data and send to the sink through other $\mathrm{CHs}$ or directly. The sink provides the communication link between the sensor network and the end-user. The advantages of using clustering technique in WSN are as follows:

1) The $\mathrm{CH}$ can prolong the battery life of the individual sensors and the network lifetime as well by implementing optimized management strategies.

2) Only one active node per cluster is necessary to guarantee complete coverage and connectivity of the network.

3) Clustering can localize the route set up within the cluster and thus reduce the size of the routing table stored at the individual sensor nodes

\section{Related Work}

Recent advances in wireless sensor networks have lead to many new protocols specifically designed for sensor networks where energy awareness is an essential consideration. But approaches like Direct Communication and Minimum Transmission Energy (MTE) [5] do not guarantee balanced energy distribution among the sensor nodes. In Direct Communication Protocol each sensor node transmits information directly to the base station, regardless of distance. As a result, the nodes furthest from the BS are the ones to die first [6]. On the other hand, in case of Minimum Transmission Energy routing protocol data is transmitted through intermediate nodes. Thus each node acts as a router for other nodes' data in addition to sensing the environment. Nodes closest to the BS are the first to die in MTE routing. So far, cluster-based technique is one of the approaches which successfully increases the lifetime and stability of whole sensor networks. We classified most important energy efficient routing techniques based on various clustering attributes like cluster formation and data gathering 
process. Figure 1 is a hierarchical diagram of different routing protocols which are widely used in WSN.

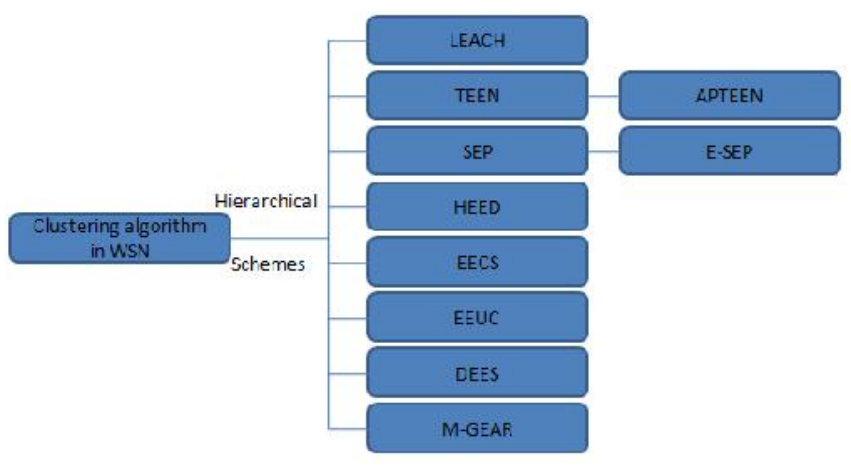

Figure 1. Classification of widely used clustering schemes in WSN

W. R. Heinzelman, A. P. Chandrakasan and H. Balakrishnan [7] proposed Low Energy Adaptive Clustering Hierarchy (LEACH) protocol in 2000. It is one of the most popular hierarchical routing algorithms for sensor networks. The idea is to form clusters of the sensor nodes based on the received strength of the signal and use local cluster heads as routers to the BS. This will save energy since the transmissions will only be done by such cluster heads rather than all sensor nodes. Optimal number of cluster heads is estimated to be 10 percent of the total number of nodes. All the data processing such as data fusion and aggregation are local to the cluster. Cluster heads change randomly over time in order to balance the energy dissipation of nodes. This decision is made by the node choosing a random number between 0 and 1 . The node becomes a cluster head for the current round if the number is less than the following threshold:

$$
T(n)=\left\{\begin{array}{c}
\frac{P}{1-P^{*}\left(r \bmod \frac{1}{P}\right)}, \quad n \in G \\
0 \quad, \quad \text { otherwise }
\end{array}\right.
$$

Where $p$ is the desired percentage of cluster heads (e.g. 0.1), $r$ is the current round and $G$ is the set of nodes that have not been cluster heads in the last $1 / p$ rounds. However the limitations of Leach protocol are that it uses single-hop routing within cluster and thus not applicable to networks deployed in large regions, dynamic clustering brings extra overhead, assumes all nodes can transmit with enough power to reach BS, if necessary (e.g., elected as CHs), each node should support both TDMA and CDMA, failure of cluster head is a problem and cluster head selection is a difficult problem to optimize. However LEACH is only effective for homogeneous network as it sets same probability of becoming cluster head to all sensor nodes. Consequently, a number of enhancements to the conventional LEACH routing protocol have been proposed and is summarized. In Centralized LEACH (LEACH-C) [8] location of the nodes is sent to the BS, which then selects $\mathrm{CHs}$ for each round. No. of $\mathrm{CH}$ is fixed to a predetermined value. The BS utilizes global knowledge of the network to produce better clusters that require less energy for data transmission.

In 2001, A. Manjeshwar and D. P. Agarwal [9] proposed Threshold sensitive Energy Efficient sensor Network Protocol (TEEN). Closer nodes form clusters, with cluster heads to transmit the collected data to one upper layer. Forming the clusters, cluster heads broadcast two threshold values. First one is hard threshold; it is minimum possible value of an attribute to trigger a sensor node. Hard threshold allow the nodes to transmit the event, if the event occurs in the range of interest. Therefore a significant reduction of the transmission delay occurs. Unless a change of minimum soft threshold occurs, the nodes don't send a new packet of data. Employing soft threshold prevents from the redundant data transmission. Since the protocol is 
to be responsive to the sudden changes in the sensed attribute, it is suitable for time-critical applications.

A. Manjeshwar and D. P. Agarwal [10] proposed Adaptive Threshold sensitive Energy Efficient sensor Network Protocol (APTEEN) protocol in 2002. The protocol is an extension of TEEN aiming to capture both time-critical events and periodic data collections. The network architecture is same as TEEN. After forming clusters the cluster heads broadcast attributes, the threshold values along with the transmission schedule to all nodes.

According to energy dissipation and network lifetime, TEEN gives better performance than LEACH and APTEEN, because of the decreased number of transmissions. The main drawbacks of TEEN and APTEEN are overhead and complexity of forming clusters in multiple levels, implementing threshold-based functions and dealing with attribute based naming of queries.

In 2004, G. Smaragdakis, I. Matta and A. Bestavros proposed Stable Election Protocol (SEP) [11]. This protocol is an extension of LEACH. It is a heterogeneous aware protocol, based on weighted election probabilities of each node to become cluster head according to their respective energy. This approach ensures that the cluster head election is randomly selected and distribution is based upon the fraction of energy of each node, which assures a uniform use of the energy. In this protocol, two types of nodes (two tier in-clustering) and two level hierarchies were considered. CHs selecting probability for normal nodes is $p_{\text {nrm }}=p_{\text {opt }} /(1+m . a)$ and for advanced nodes $p_{a d v}=p_{\text {opt }}(1+a) /(1+m . a)$, where $p_{\text {opt }}$ is the optimal probability of each node to become $\mathrm{CH}$. The idea is that the advance nodes have to become the $\mathrm{CHs}$ more often than normal nodes. SEP gives better result as the value of $a$ and $m$ will increase.SEP maintains the constraints of well-balanced energy consumption. As initially, advanced nodes have to become the $\mathrm{CHs}$ more often than normal nodes. Thus, SEP yields longer stability region by utilizing the extra energy of more powerful nodes. But the main drawback of SEP method is that the election of the cluster heads among the two type of nodes is not dynamic, which results that the nodes that are far away from the powerful nodes will die first. SEP sets two probabilities based on only nodes initial energy. But the possibility in SEP is that after certain rounds an advanced node might become normal node due to more energy consumption. In such conditions, SEP selects low energy node as a maximum probability of being cluster head as SEP is only aware of nodes initial energy.

The extension of SEP, Femi A. Aderohunmu and Jeremiah D. Deng proposed E-SEP [12] in the year of 2009.E-SEP considers three types of nodes, normal nodes, intermediate nodes and advance nodes. Where, advance nodes are in a fraction of total nodes with an additional energy as in SEP and a fraction of nodes with some extra energy greater than normal nodes and less than advance nodes, called intermediate nodes, while rest of the nodes are normal nodes. As in SEP, the initial energy for normal nodes is $E_{0}$, and for advanced nodes is $(1+) \cdot E_{o}$. E-SEP added another set of initial energy nodes i.e. $E_{\text {int }}$ as $(1+) \cdot E_{o}$, where $=a / 2$. Like SEP, in E-SEP CHs are selected depending on probability of each type of node. However, energy dissipation is controlled to some extent due to three levels of heterogeneity. ESEP has same drawbacks as SEP. For selecting $\mathrm{CHs}$ it also set the probability based on nodes initial energy. E-SEP also does not consider residual energy of nodes. Other extensions of SEP protocols are ASEP-E [13], Z-SEP [14], T-SEP [15] and H-SEP [16]. In ASEP-E, four types of nodes have been considered for assigning the probability of each type of nodes. The new type of nodes are referred to 'super advance nodes' whose initial energy is $(1+b)^{\star} E_{o}$, where $b=a / 4$. Z-SEP is zone based clustering algorithm where the advance nodes only have the probability to become a cluster head. Drawbacks of Z-SEP are, nodes cannot be deployed randomly and only advance nodes are selected as a cluster head. As a result advance nodes are died soon. TSEP is threshold based SEP, uses three level of heterogeneity. As in T-SEP the CHs selection is done based on threshold value, it decreases the throughput due to threshold sensitivity. $\mathrm{H}$ SEP is heterogeneous aware protocol to minimize transmission cost between $\mathrm{CH}$ and $\mathrm{BS}$. In $\mathrm{H}$ SEP selection of cluster heads cannot be done in a distributive way.

In 2004, O Younis, S Fahmy proposed HEED: A hybrid, energy-efficient, distributed clustering approach [17]. HEED is a multi-hop clustering algorithm for wireless sensor networks, with a focus on efficient clustering by proper selection of cluster-heads based on the physical distance between nodes. Cluster construction in HEED Reference 2 is performed based on two parameters the node's residual energy, and intra-cluster communication cost. In HEED, elected $\mathrm{CHs}$ have relatively high average residual energy. Moreover HEED aims to provide evenly

Energy Efficient Clustering for Wireless Sensor Networks using EASSR (Y.Chalapathi Rao) 
distributed $\mathrm{CHs}$ throughout the network. $\mathrm{CHs}$ send the aggregated data to the $\mathrm{BS}$ in a multi-hop fashion rather than single-hop fashion of LEACH. Similar to LEACH, the performing of clustering in each round imposes significant overhead in the network. This overhead causes noticeable energy dissipation which results in decreasing the network lifetime. The use of tentative $\mathrm{CHs}$ that do not become final $\mathrm{CH}$ leave some uncovered nodes. As per HEED implementation, these nodes are forced to become a $\mathrm{CH}$ and these forced $\mathrm{CH}$ s may be in range of other $\mathrm{CH}$ or may not have any member associated with them. As a result, more $\mathrm{CHs}$ are generated than the expected number and this also accounts for unbalanced energy consumption in the network.

In 2005, M. Ye, C. Li, G. Chen and J. Wu [18] proposed Energy Efficient Clustering Scheme (EECS) protocol. It is novel clustering scheme for periodical data gathering applications for wireless sensor networks. It elects cluster heads with more residual energy through local radio communication. In the cluster head election phase, a constant number of candidate nodes are elected and compete for cluster heads according to the node residual energy. The competition process is localized without iteration. Further in the cluster formation phase, a novel approach is introduced to balance the load among all cluster heads. But on the other hand, it increases the requirement of global knowledge about the distances between the cluster-heads and the base station.

In 2005, C. Li, M. Ye, G. Chen and J. Wu proposed An energy-efficient unequal clustering mechanism for wireless sensor networks [19]. EEUC is designed for periodic data gathering applications in WSN. According to this scheme the nodes in one region compete to become $\mathrm{CH}$ in such a way that the node's competition range decreases as its distance to the base station decreasing. Thus the nodes closer to the BS consume less energy for intra cluster routing and can utilize it for inter-cluster routing. Energy consumed by cluster heads per round in EEUC much lower than that of LEACH standard but similar to HEED protocol.

In 2006, Q. Li, Z. Qingxin and W. Mingwen [20] proposed Distributed Energy Efficient Clustering Protocol (DEEC) protocol. This protocol is a cluster based scheme for multi level and two level energy heterogeneous wireless sensor networks. In this scheme, the cluster heads are selected using the probability based on the ratio between residual energy of each node and the average energy of the network. The epochs of being cluster- heads for nodes are different according to their initial and residual energy. The nodes with high initial and residual energy have more chances to become the cluster heads as compared to nodes having low energy. The main disadvantage of DEEC is advanced nodes are always penalized, particularly when their residual energy reduced and become in the range of the normal nodes. In this position, the advanced nodes die rapidly than the others.

In Gateway-Based Energy-Aware Multi-Hop Routing Protocol for WSNs: M-GEAR [21] proposed in 2013, sensor nodes are divided into four regions based on nodes location. Base Station is situated out of sensing area and a special node termed as Gateway is placed at the Centre position. Nodes which are near to BS or Gateway use direct communication to send packets directly to BS or Gateway. Rest nodes are divided into two equal regions, and only these regions are taking part of CHs formation exactly the same way as of LEACH. M-GEAR performs better than LEACH but it has certain limitations. M-GEAR is a region based protocol where node has to decide whether to take part in clustering or in direct communication which increase overhead

\section{Energy Aware Sleep Scheduling Routing Scheme in WSN (EASSR)}

EASSR is an Energy Aware Sleep Scheduling Routing Scheme for Wireless Sensor Networks. The main idea of EASSR is to minimize energy consumption in order to enhance networks stability and lifetime. EASSR uses systems average residual energy per round and energy consumption rate in previous round to select cluster heads. The model is described as follows.

\subsection{Network Model}

In EASSR protocol following network assumptions is considered:

a) All sensor nodes in the networks are static and energy is limited. We assume that a fraction of the total nodes are equipped with more energy. Let $m$ be fraction of the total nodes $\mathrm{n}$, which are equipped with a time more energy than the other nodes. 
We refer these nodes as advance nodes and (1-m) $x n$ are normal nodes. So, nodes have different initial energy and the battery could not be rechargeable.

b) All nodes are stationary once deployed randomly in the field and they are left unattended after deployment.

c) For simplicity and convenience, the sensing mode is Boolean mode.

d) All nodes should be roughly time synchronized on the order of seconds.

e) Nodes are location-unaware, i.e. not equipped with GPS-capable antennae.

f) There is single BS located in the centre of the field. The BS is a stationary, highenergy node; position of the base station is fixed.

g) Each sensor node periodically senses the monitored environment, and has a perpetual desire to send the sensed data to the BS. Sensor nodes are probed with power control capabilities to change their transmitted power.

h) Radio transmission in all directions has the same amount of energy consumption.

i) The nodes are considered to die only when their energy is exhausted.

\subsection{Energy Model}

Currently, there is a great deal of research in the area of low-energy radios. Different assumptions about the radio characteristics, including energy dissipation in transmit and receive modes, will change the advantages of different protocols. In our work, first order radio model [22] is adopted for measuring energy consumption by sensor nodes while communicating. We assume a simple model where the radio dissipates $E_{\text {elec }}=50 \mathrm{~nJ} / \mathrm{bit}$ to run the transmitter or receiver circuitry and $\epsilon_{a m p}=100 \mathrm{pJ} / \mathrm{bit} / \mathrm{m}^{2}$ for the transmit amplifier to achieve an acceptable S-N ratio. To transmit a $k$-bit message a distance $d$ using our radio model, the radio expends:

$$
\begin{aligned}
& E_{T X}(k, d)=E_{T X-\text { elec }}(k)+E_{T X \text {-amp }}(k, d) \\
& E_{T X}(k, d)=E_{\text {elec }} * k+\epsilon_{\text {amp }} * k * d^{2} \\
& E_{T X}(k, d)=E_{\text {elec }} * k+\epsilon_{f s} * k * d^{2}, \text { if } \quad d \leq d_{0} \\
& =E_{\text {elec }} * k+\epsilon_{m p} * k * d^{4}, \text { if } \quad d \succ d_{0}
\end{aligned}
$$

In wireless transmission, attenuation of sending power decreased exponentially with the increasing transmission distance. Equuation (1) represents the amount of energy consumed for transmitting $k$ bits of data to $d$ distance. Equation (3) represents the amount of energy consumed for receiving $k$ bits of data which is caused only by circuit loss. To receive $k$ bit message, the radio expends:

$$
\begin{aligned}
& E_{R X}(k)=E_{R X-\text { elec }}(k) \\
& E_{R X}(k)=E_{\text {elec }} * k
\end{aligned}
$$

Here $d$ refers the transmission distance i.e. distance between a member-node and its cluster-head or between cluster-head and base station (BS); $d_{0}$ is threshold distance; $E_{\text {elec }}$ the energy consumption per bit in the transmitter and receiver circuitry by a node hen $d \leq d_{0}$ and $d>$ $d_{0}$, where $d_{0}=\sqrt{\frac{\epsilon_{f s}}{\epsilon_{m s}}}$.

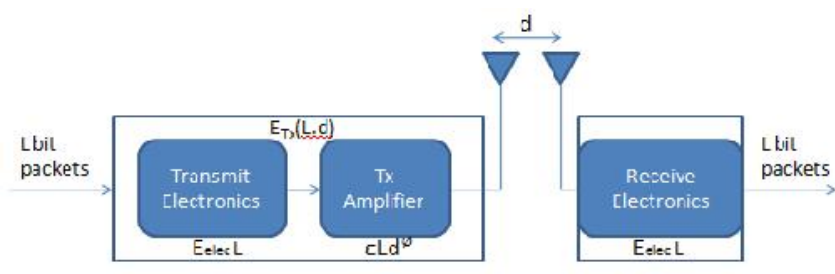

Figure 2. Energy Dissipation model 
$\epsilon_{f s}$ and $\epsilon_{m p}$ are the energy consumption coefficient of the amplifier, when the transmission distance is less than the threshold distance; free space channel model is used. On the contrary, Multi-path fading channel model is used present in Equation (2). In addition to transmission and reception of data, data aggregation performed at $\mathrm{CHs}$ needs a significant amount of energy (EDA). Energy Dissipation Model is shown in Figure 2.

\subsection{EASSR Protocol}

Proposed scheme is a distributed energy aware clustering algorithm. It considers both system average energy and residual energy information of nodes. Similar to that of various energy efficient protocols like LEACH, SEP, E-SEP, DEEC our proposed scheme also continues with round and each round can be divided into initialization stage and stable working stage. Starting of a round, BS broadcasts HELLO packets among the sensors periodically. If the $\mathrm{RSSI}$ of the received signal is greater than clustering threshold then no need to form clusters. Based on stronger RSSI (Receiving Signal Strength Indicator) nodes closer to the BS are selected to send their data directly to BS. This region is called direct communication region. Rest of the nodes follow dynamic clustering technique.

So, EASSR uses two techniques to transmit data to base station. Techniques are:

a) Direct communication: Nodes in this zone send their data directly to base station. Nodes sense environment gathers data or information and send it directly to base station.

b) Transmission via Cluster head: Nodes in this zone transmit data to base station through clustering. Cluster head is selected among nodes and organize themselves into small groups known as clusters. Then Cluster head collect data from member nodes, aggregate it and transmit it to base station. Cluster head selection is most important. But before performing cluster formation we introduce sleep-awake policy for the sensors.

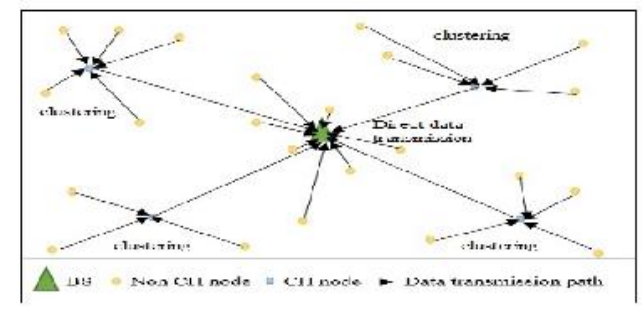

Figure 3. Network Architecture

In WSN due to limited resources and vulnerable nature of individual sensor, sensors are deployed with high density. As a result same area is covered by many sensor nodes.

\subsection{Node Pairing}

Before performing clustering a node has to select its nearest node. A node sends a request message Find Nearest Neighbour. The 1-hop neighbour nodes which are closer to that node send a reply with their distances from that particular node and they are included in Eligible Neighbour List. Then the node in the Eligible Neighbour List which has maximum RSSI range of the received signal is selected as next node. The two nodes are added as coupled and then Node Paired ID message is broadcast in the network. Algorithm 3 presents the mechanism of node pairing.

\subsection{Cluster Heads Selection}

In order to effectively deal with the heterogeneous energy capacities in sensor networks, it is obvious that higher the energy a node has, the higher is the probability of it to become $\mathrm{CH}$. The nodes exceeding average energy level, higher probability to become $\mathrm{CH}$ nodes than the nodes having residual energy below the system average energy level. If the energy of $\mathrm{CH}$ is too low, then after receiving information from a handful of its member nodes, 
$\mathrm{CH}$ may die because of low or dead battery. In such circumstances the information that the $\mathrm{CH}$ node has received before it becomes dead, also gets lost. To minimize the data loss EASSR elects a node as a cluster head based on the decision that, the remaining energy of that sensor node is greater than system average energy. Decisions are made by node based on energy level shown in Algorithm 2. Algorithm 5 gives the clear picture of selection appropriate cluster head. At the starting of cluster head selection phase BS receives the status of the current energy level from all sensor nodes in the network. Then selections of powerful nodes are done based on the received energy values. The BS computes the average energy level of the active nodes as follows:

$$
E_{\text {avg }}=\frac{\sum E_{\text {resi }}}{m}
$$

Where $m$ is the total number of active nodes $(\leq N) ; E_{\text {resi }}$ is nodes residual energy.

So, EASSR protocol uses $E_{\text {avg }}$ to be the main parameter for selecting $\mathrm{CHs}$ and handle well the heterogeneous energy capacities among the sensor nodes considering system average energy in each round. After BS broadcasts average energy of the network, node having remaining energy greater than or equal to the system average energy include themselves in the set of eligible cluster heads. If a node finds its $E_{\text {resi }} \geq E_{\text {avg }}$ then it sends a request message to find eligible neighbours. The 1-hop neighbour nodes which are closer to that node send a reply with their Energy Consumption Rate $E_{e c r}$ in previous round. The node which has minimum energy consumption rate in previous round and with $E_{\text {resi }} \geq E_{\text {avg }}$ is selected as $\mathrm{CH}$ node, where energy consumption rate is as follows:

$$
E_{e c r}=\frac{E_{0}-E_{r e s i}}{r-1}
$$

In order to assure average number of $\mathrm{CHs},(P X N)$ number $\mathrm{CHs}$ are selected for $\mathrm{N}$ number of alive nodes at each round. $\mathrm{P}$ is the desired percentage of $\mathrm{CHs}$ per round.

\subsection{Data Transmission and Data Aggregation}

In this phase, all nodes in Active-mode transmit their sensed data to $\mathrm{CH}$ during their assigned TDMA slots. Nodes in Sleep-mode do not participate and thus save their energy by turning their transceiver off. The selected $\mathrm{CHs}$ broadcast, broadcasts its Cluster head advertisement message. All non-Cluster head active nodes, sends joining request message to that cluster head, from which it received the highest RSSI. Cluster head accepts the joining request and forms respective clusters. Then $\mathrm{CHs}$ aggregate received data from each node and transmit to BS. Data aggregation may be considered to be an effective technique to compress the amount of data sent to BS. Due to data aggregation technique a noticeable amount of energy is saved. If there are $N$ total numbers of nodes and $X$ is the optimal number of $\mathrm{CHs}$ then the average number of nodes in each cluster will be:

$$
\frac{N}{X}-1
$$

In order to transmit data, the radio of a non- $\mathrm{CH}$ node dissipates $E_{T X}$ to run the transmitter circuitry and $E_{a m p}$ for transmit amplifier to achieve acceptable SNR (Signal-to-Noise Ratio). So, for transmission of $k_{c}$ bit message a non- $\mathrm{CH}$ node expands following the first order radio model.

$$
E_{\text {non }-\mathrm{CH}}=\left(\frac{N}{X}-1\right)\left(E_{T X} * k_{c}+\epsilon_{\text {amp }} * k_{c} * d_{t o C H}^{2}\right)
$$

Where $d_{t o C H}^{2}$ is the distance between nodes and $\mathrm{CHs}$. 
To receive data from non- $\mathrm{CH}$ node by the radio of $\mathrm{CH}$ in each cluster expands:

$$
E_{\text {receive }}=\left(E_{R X} * k_{c}\right)\left(\frac{N}{X}-1\right)
$$

Where, $E_{R X}$ is energy dissipated by receiver circuitry for receiving data. Energy dissipated by $\mathrm{CH}$ to aggregate data received from its associated nodes.

$$
E_{\text {avg }}=\left(E_{D A} * k_{c}\right)\left(\frac{N}{X}\right)
$$

Transmission energy $E_{T}$ dissipated by $\mathrm{CH}$ to transmit aggregated data to the $\mathrm{BS}$ is:

$$
E_{T}=\left(E_{T X} * k_{A}+\in_{\text {amp }} * k_{A} * d_{\text {toBS }}^{2}\right)
$$

Where $k_{A}$ is aggregated data and $d_{t o B S}^{2}$ is the distance between $\mathrm{CH}$ and BS. Total energy dissipated by $\mathrm{CH}$ in a round is:

$$
E_{C H}=E_{\text {receive }}+E_{\text {avg }}+E_{T}
$$

Total energy dissipated by $\mathrm{CH}$ is the energy dissipated in reception of data from its associated nodes shown in Equation (8), aggregation of received data shown in Equation (9) and transmission of that data to the BS shown in Eq. (10). After performing aggregation each $\mathrm{CH}$ sends concise data to the BS. below.

Algorithm of Energy Aware Sleep Scheduling Routing Protocol (EASSR) is depicted

1. SensingRegionSelection(): This algorithm determines the region where clustering is required.

Input: Position of the sensor nodes and BS.

Output: Direct transmission region, clustering region.

begin

for a given simulation time $T$ do

$B S$ broadcasts HELLO packets periodically.

if a sensor node listen HELLO packet then

Reply with residual energy.

if the $R S S I$ of the received signal $\geq R S S I_{c h}$ then

No need to form clusters.

else

End

Clustering occurs according to ClusterSelection()

2. ClusterSelection(): This algorithm grouped the sensor nodes into clusters.

Input: No. of Sensor nodes, Initial node energy, probability (p), No of rounds.

Output: Cluster heads, Clusters.

begin

if any node is alive then

follow nodePairing() to pair with closest neighbour.

follow nodeClassStatusSetup() to decide about the active nodes.

if Node.Class = ACTIVE for any node then

for each round do

$\mathrm{BS}$ choose $\mathrm{CH}$ s following $\mathrm{CH}$ setup()

if a node is Cluster Head then

Broadcasts its $\mathrm{CH}$ advertisement message

TELKOMNIKA Vol. 15, No. 3, September 2015 : 554 - 568 
All non- $\mathrm{CHs}$ active nodes, sends joining request message to that $\mathrm{CH}$, from which it received the highest $R S S I$.

Cluster head accepts the joining request.

Forms respective clusters.

End

3. NodePairing(): This algorithm coupled the nodes.

Input: No. of Sensor nodes, RSS

Output: Set of paired-node

begin

A node broadcasts a request message Find_Closest_Neighbour to its neighbours.

The neighbours respond reliably.

The neighbour with the strongest RSSI is selected as the next node.

Node_Status $=$ PAIRED

End

Broadcast Node_Paired_ID message

4. NodeClassStatusSetup(): This algorithm set the mode of node whether switch to sleep mode or active mode for this round.

Input: No. of Sensor nodes, Node residual energy.

Output: Sleep node, Active node.

begin

Get $N$ paired node sets in the network.

if node $==$ PAIRED then

for each pair set do

Node broadcasts an Energy Msg message to its neighbouring node.

Receiving Energy Msg from its neighbouring node, updates its own Neighbour_Table.

if $E(r)_{\text {node }}>E(r)_{\text {neighbour }}$ then

Set Node. Class $=$ ACTIVE and Node. Status $=1$ else

The node Broadcasts go_To_Sleep message to neighbours.

Node. Class $=$ SLEEP

Set Node. Status $==0$

Nodes receive their status update

for a pair do

if neighbour node is dead then

Another node of that pair awake for remaining rounds.

else Node.Class = ACTIVE for whole networklife time.

End

5. CHsetup(): This algorithm select the powerful nodes as cluster head

Input: No. of Sensor nodes, Initial node energy, Node Residual Energy, Energy Consumption rate, No of rounds.

Output: Cluster heads.

begin

$B S$ receives the status of the $E_{\text {resi }}$ from all nodes.

Then based on this $B S$ computes $E_{\text {avg }}$ of the active nodes.

Broadcasts the $E_{\text {avg }}$

for each node having $E_{\text {resi }} \geq E_{\text {avg }}$ do

Include in the set of eligible $\mathrm{CHs}$

if a node finds its $E_{\text {resi }} \geq E_{\text {avg }}$ then

it sends a request message to find eligible neighbours

The 1-hop neighbour nodes closer to that node send a reply with their $E_{e c r}$ of $r-1$.

The node which has min $E_{e c r}$ and with $E_{r e s i} \geq E_{a v g}$ is selected as $\mathrm{CH}$ node.

End 


\section{Simulation Results}

In this section we compare the performance of LEACH, SEP and M-GEAR under two level heterogeneous network systems. For simulations we have considered $m=0.3$ and $a=3$ i.e. 30 percent of nodes as advance nodes with energy factor 3 (i.e. equipped with 3 times more energy than that other normal nodes).

Simulation result shows that EASSR significantly prolongs the stable region compared to that of LEACH, SEP and M-GEAR. Nodes of LEACH have short instability period compared to M-GEAR, SEP and our protocol. But at the end the LEACH performs better than SEP. The reason behind this is, $\mathrm{LEACH}$ sets equal probability for selecting $\mathrm{CHs}$ for all normal and advance nodes. As a result the advance nodes die slowly, thus increasing slightly unstable region. On the other hand, SEP sets high probability for advance nodes to become CHs. But SEP considers nodes initial energy only to select $\mathrm{CHs}$ and after a few rounds an advance node might become normal node due to more energy consumption. In such conditions there is probability that SEP selects low energy node as cluster head with high probability. Because of this unstable region in SEP is not longer than our protocol (EASSR). In M-GEAR, the sensing field is divided into regions to communicate directly with BS or gateway and as M-GEAR does not take care of advance node so all nodes die quickly. In case of our protocol, we put some nodes to sleeping mode that preserve some amount of energy at each round. And here $\mathrm{CH}$ selection is based on energy consumption Rate and average system energy. That's why EASSR achieves maximum stability period compared to others. Simulation results are shown in Figure 4.

From Figure 4 when there are 30 percent advance nodes with additional energy factor $\mathrm{a}=3$, first node dies in LEACH after 1035 rounds, in M-GEAR after 890 rounds, in SEP after 1379 rounds and in EASSR after 1402 rounds respectively. So, EASSR performs 1.35 times better than LEACH, 1.58 times better than M-GEAR and 1.02 times better than SEP. If we study the graph we can see that our protocol not only performs better than the other three, but distribution of energy consumption is also uniform. As in each round EASSR checks for system remaining energy and system average energy, so cluster head selection is done in a proper way by ensuring that $\mathrm{CH}$ has comparatively higher energy than rest of nodes. In Figure 5 we analyze the number of $\mathrm{CHs}$ selected every round under $\mathrm{m}=0.1, \mathrm{a}=1$ parameter settings.

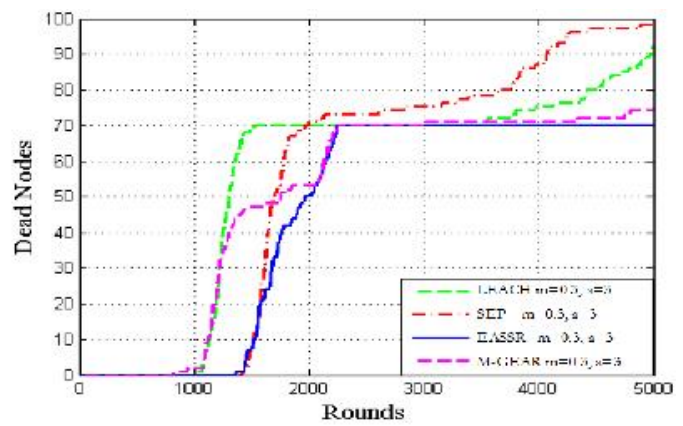

Figure 4. Dead nodes vs. Rounds ( $m=0.3$, $a=3)$

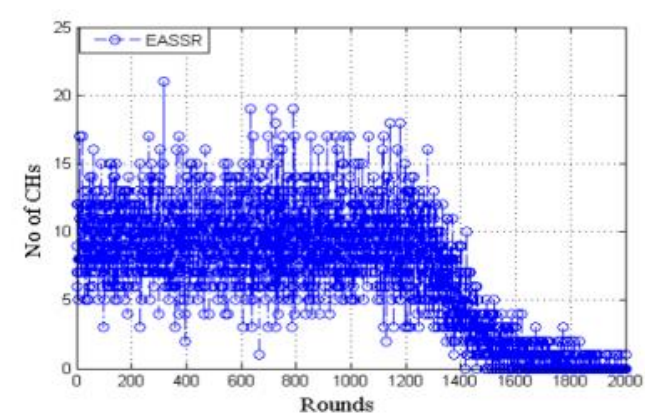

Figure 5. No. of cluster heads vs. Rounds in $\operatorname{EASSR}(m=0.1, a=1)$

\subsection{Network Lifetime}

In Figure 6, we show the results of network lifetime. Nodes are considered dead after consuming initial energy. EASSR protocol obtains the longest network lifetime and unstable region among the other three. This is because, here the energy consumption is well distributed among nodes. Network is divided in EASSR into two logical regions and it balances energy consumption among sensor nodes and some nodes are put to off mode in each round to save energy without losing data. When there are 10 percent of advance node with factor $a=1$, last node dies in LEACH after 1970 rounds, in M-GEAR after 3800 rounds, in SEP after 3666 rounds and in our protocol (EASSR) after 4110 rounds. So EASSR outperforms 2.08 times than LEACH, 1.08 times than M-GEAR and 1.12 times than SEP. So, the network life for EASSR is increased as compared to others. 


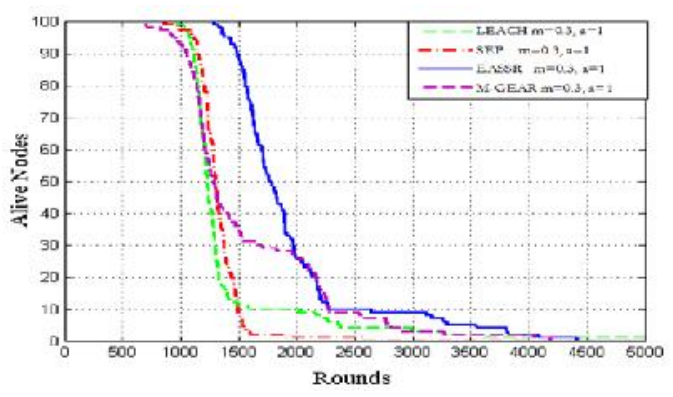

Figure 6. Alive nodes vs. Rounds ( $m=0.1, a=1$ )

\subsection{Throughput}

We study packet delivery fraction (throughput) in Figure 7 and Figure 8 under two types of parameter settings as mentioned above. The throughput of EASSR is higher than LEACH and SEP. This means that EASSR guarantees a proper distribution of cluster head and cluster heads in more rounds. These cluster heads will report to the base station hence throughput increases. On the other hand the throughput of M-GEAR is much higher than all due to direct communication nodes. Thus nodes transmit more messages to the base station. Due to direct communication of M-GEAR, cluster heads do not get the opportunity to aggregate information. This leads to data redundancy. It is worth noticing that our protocol employs sleep scheduling method to send concise and important data in a way which minimizes data redundancy.

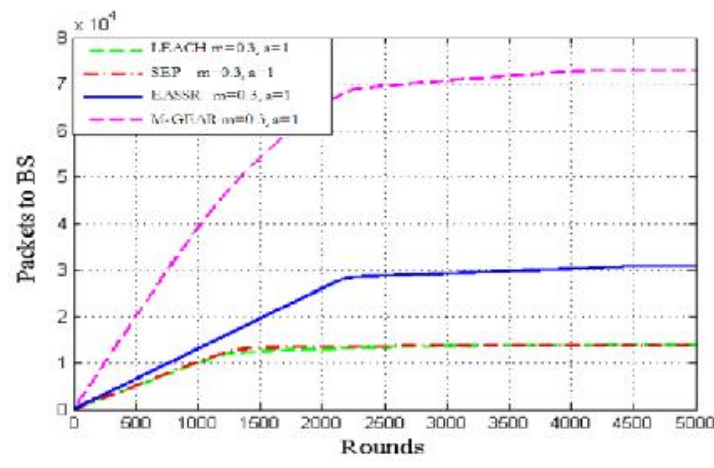

Figure 7. Throughput vs. Rounds $(m=0.1, a=1)$

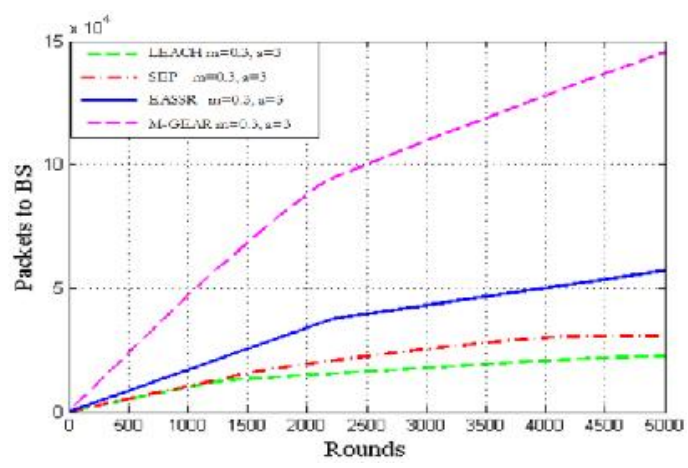

Figure 8. Throughput vs. Rounds ( $m=0.3, a=3$ )

\subsection{Residual Energy}

Figure 9 average residual energy of network per round and Figure 10 show energy consumption rate per round. We assume that a normal node has 0.5 joule energy initially and each of $m \%$ advance nodes has factor of a time more energy. As we consider three parameter settings, total system energy varies. When $m=0.3 a=3$ : The total energy of 100 node network is 95 joule.

Our proposed protocol EASSR yields minimum energy consumption with respect to LEACH, SEP, M-GEAR. From the result it is clearly depicted that our protocol out-performs these three protocols in terms of energy consumption per round. M-GEAR shows slightly better result than LEACH and SEP due to deployment of gateway node and sending data via this to BS. But to deploying gateway node is cost effective, where as EASSR selects $\mathrm{CHs}$ considering energy consumption rate in previous round. So the distribution of energy consumption in EASSR is done in an equitable fashion. 


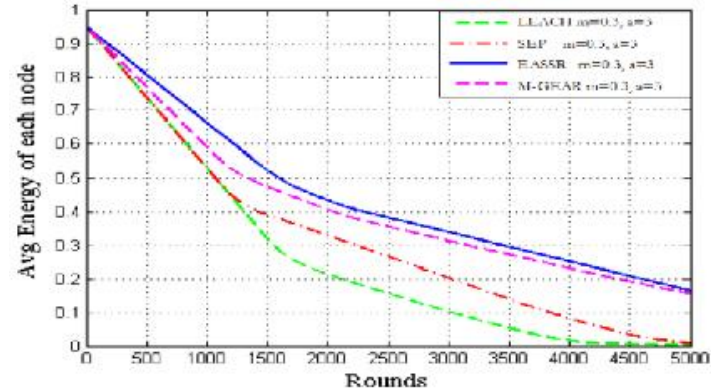

Figure 9. Average Energy vs. Rounds ( $m=0.3$, $a=3)$

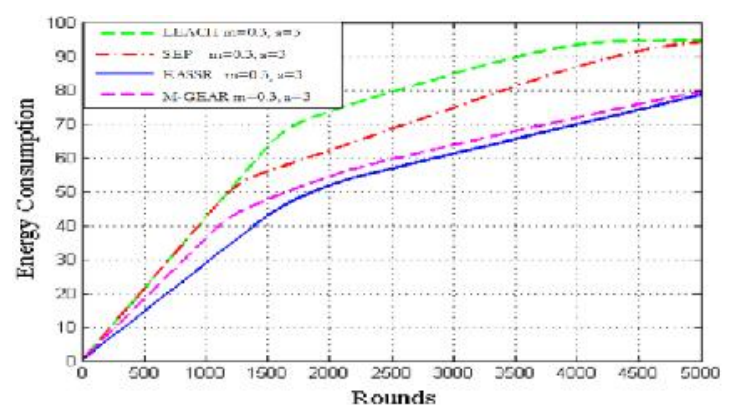

Figure 10. Energy Consumption Rate vs. Rounds $(m=0.3, a=3)$

\subsection{Performance Evolution fewer than Three Level of Heterogeneity}

We also examine the sensitivity of EASSR to the higher degree of heterogeneity in the network. We compare our protocol with Enhanced-SEP which introduced three level of heterogeneity \{advance, normal and intermediate nodes in sensor field. We set the heterogeneity parameters to $m=0.2 b=0.3$ and $a=3$ i.e. $20 \%$ and $30 \%$ of the nodes be advanced and intermediate nodes with additional energy levels: $a=3$ and $a=1.5$ respectively. Total number of rounds considered is 10000 . E-SEP obviously shows better results than SEP, LEACH and M-GEAR due to availability of more nodes with extra energy. To have a fair comparison we examine our protocol under equal energy distribution and compare with E-SEP. First node and last node die in E-SEP after 1350 and 5100 rounds respectively. On the other hand the first node of our proposed protocol die after 1350 rounds and last node die after 8900 rounds, thereby achieving greater instability period as compared to E-SEP protocols. Results shows that EASSR increases the network lifetime and performs 1.75 times better than E-SEP (see Figure 11). EASSR also increases the no. of packets sent to BS as shown in Figure 12.

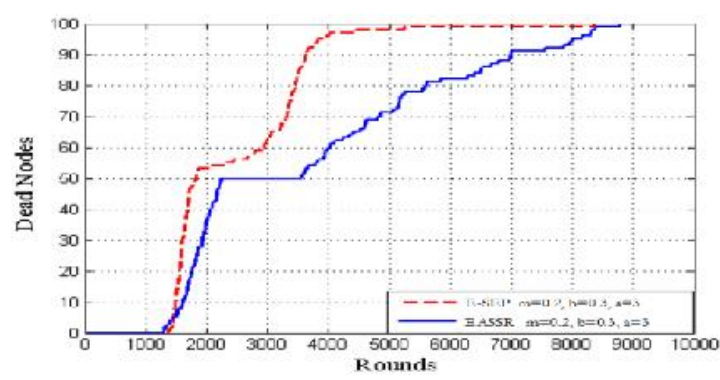

Figure 11. Dead nodes vs. Rounds ( $m=0.2$, $b=0.3, a=3$ )

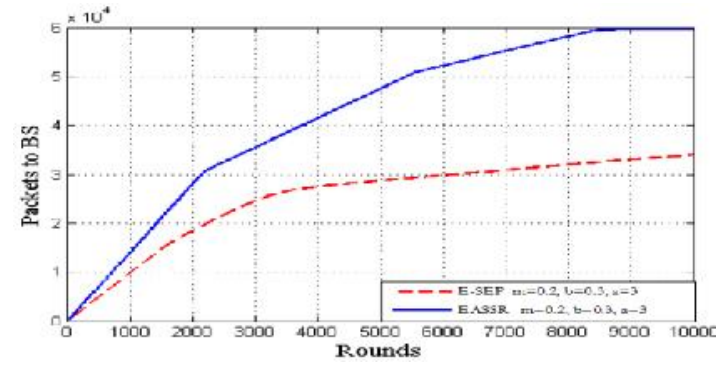

Figure 12. Throughput vs. Rounds ( $m=0.2$, $b=0.3, a=3$ )

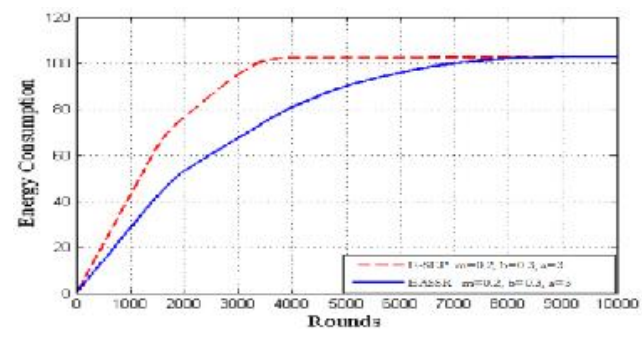

Figure 13. Energy Consumption Rate vs. Rounds ( $m=0.2, b=0.3, a=3$ ) 
It performs better than E-SEP because of switching between alive and sleep mode in nodes. So, we get information for longer period of time. Figure 13 shows clearly that EASSR yields minimum energy consumption despite increasing energy heterogeneity level.

\section{Conclusion and Future Scope}

In wireless sensor networks, heterogeneous nodes increase networks complexity. Most clustering algorithms based on network heterogeneity perform in respect of nodes initial energy. But it is obvious that over time a homogeneous network also becomes heterogeneous with nodes of unequal remaining energy. On the other hand, over rounds an initially set advance node can become a normal node and vice versa. Unlike SEP, EASSR considers this type of situation. EASSR performs equally well for homogeneous network as well as heterogeneous network. In our approach, nodes are selected as a cluster head based on their residual energy and systems average energy. Nodes having more or equal energy than systems average energy always have higher chances to become a cluster head, so the distribution of energy consumption is done in an equitable fashion. In EASSR nodes switch between sleep and active modes in order to minimize energy consumption. We showed that EASSR can better adapt the applications with great heterogeneous energy capacities in sensor networks. In our proposed strategy, stability period of network, life time of network, data rate (throughput) and remaining energy with respect to rounds has been optimized. Simulation results show that there is significant improvement in all these parameters when compared with some of the existing routing protocols e.g., SEP, LEACH, E-SEP and M-GEAR.

\section{References}

[1] K Akkaya, M Younis. A survey on routing protocols for wireless sensor networks, ad hoc networks. Ad Hoc Networks, CiteSeerX. 2005; 3: 325-349.

[2] SS Kanhere, $\mathrm{N}$ Ahmed, $\mathrm{S}$ Jha. The holes problem in wireless sensor networks: a survey. ACM SIGMOBILE Mobile Computing and Communications Review. 2005; 9(2): 4-18.

[3] V Pal, G Singh, RP Yadav. SCHS: Smart cluster head selection scheme for clustering algorithms in wireless sensor networks. Wireless Sensor Network journal, Scientific Research. 2012; 4: 273-280.

[4] R Rajagopalan, PK Varshney. Data aggregation techniques in sensor networks: a survey. IEEE Communications Surveys and Tutorials. 2006; 8(4): 48-63.

[5] TJ Shepard. Channel access scheme for large dense packet radio networks. In Proc. ACM SIGCOMM. 1996; 26: 219-230.

[6] AP Chandrakasan, WR Heinzelman, H Balakrishnan. Energy-efficient communication protocol for wireless micro-sensor networks. in Proceedings of the $33^{\text {rd }}$ Hawaii International Conference on System Sciences. 2000.

[7] W Heinzelman, A Chandrakasan, H Balakrishnan. Application-specific protocol architecture for wireless micro-sensor networks. Proc. 33rd Hawaii Int', I. Conf. Sys. Sci. 2000: 660-670.

[8] SD Muruganthan, DCF Ma, B Rollyi, A Fapojuwo. A centralized energy-efficient routing protocol for wireless sensor networks. IEEE Radio Communications. 2005; 43(3): 8-13.

[9] A. Manjeshwar and D.P. Agarwal. TEEN: a routing protocol for enhanced efficiency in wireless sensor networks. In 1st International Workshop on Parallel and Distributed Computing Issues in Wireless Networks and Mobile Computing, April 2000, pp.2009-2015.

[10] A Manjeshwar, DP Agarwal. APTEEN: A hybrid protocol for efficient routing and comprehensive information retrieval in wireless sensor networks. Parallel and Distributed Processing Symposium, Proceedings International, IPDPS. 2001.

[11] G Smaragdakis, I Matta, A Bestavros. SEP: A stable election protocol for clustered heterogeneous wireless sensor networks. In Second International Workshop on Sensor and Actor Network Protocols and Applications (SANPA). 2004.

[12] FA Aderohunmu, JD Deng. An enhanced stable election protocol (sep-e) for clustered heterogeneous wsn. Department of Information Science, University of Otago. 2009.

[13] A Kaur, S Singh, K Singh. Advanced SEP-E protocol for extending the lifetime of the heterogeneous wireless sensor networks. International Journal of Networks and Systems. 2013; 2: 27-33.

[14] S Faisal, N Javaid, A Javaid, MA Khan, SH Bouk, ZA Khan. Zonal-stable election protocol for wireless sensor networks. Journal of Basic and Applied Scientific Research. 2013; 3: 132-139.

[15] A Kashaf, N Javaid, ZA Khan, IA Khan. T-SEP: Threshold-sensitive stable election protocol for wsns. Frontiers of Information Technology (FIT) of IEEE. 2012: 164-168.

[16] AA Khan, N Javaid, U Qasim, Z Lu, ZA Khan. HSEP: Heterogeneity-aware hierarchical stable election protocol for wsns. Seventh International Conference on Broadband, Wireless Computing, Communication and Applications. 2012. 
[17] O Younis, S Fahmy. HEED: A hybrid, energy-efficient, distributed clustering approach for ad-hoc sensor networks. IEEE Trans. Mobile Comput. 2004; 3: 366-379.

[18] M Ye, C Li, G Chen, J Wu. EECS: an energy efficient cluster scheme in wireless sensor networks. In IEEE International Workshop on Strategies for Energy Efficiency in Ad Hoc and Sensor Networks (IEEE IWSEEASN-2005). Phoenix, Arizona. 2005.

[19] C Li, M Ye, G Chen, J Wu. An energy-efficient unequal clustering mechanism for wireless sensor networks. In IEEE International Conference on Mobile Ad-hoc and Sensor Systems. 2005: 604-611.

[20] Q Li, Z Qingxinand, W Mingwen. Design of a distributed energy efficient clustering algorithm for heterogeneous wireless sensor networks. Computer Communications. 2006; 29(12): 2230-2237.

[21] Q Nadeem, MB Rasheed, N Javaid, ZA Khan, Y Maqsood, A Din. M-GEAR: Gateway-based energyaware multi-hop routing protocol for wsns. Broadband and Wireless Computing, Communication and Applications (BWCCA) of IEEE. 2013: 164-169.

[22] SK Mitra, MK Naskar. Comparative study of radio models for data gathering in wireless sensor network. International Journal of Computer Applications. 2011; 27(4): 49-57. 